\title{
Review of Reports of Curing Cancer with CellSonic VIPP Machines
}

\author{
Karem Belal $^{1^{*}}$ \\ ${ }^{1}$ Professor Dr. Karem Belal, MbChB, FRCS, MRCGP
}

Received: November 07, 2017; Accepted: November 22, 2017; Published: December 22, 2017

*Corresponding author: Professor Dr. Karem Belal, MbChB, FRCS, MRCGP, Paphos, Cyprus, E-mail: ksbelal@hotmail.com

\section{Introduction}

In 2016, Andrew Hague, President of CellSonic Limited and a Professor of Advanced Medicine discovered that cancer can be cured by applying the very intense pulses of the CellSonic medical machine. His logic was based on an assessment of safety for he insisted that there must be no risks to the patient, the operator and the environment. He then reasoned that the pressure of the pulses would stop the cancer based on published hypotheses and a report from Innsbruck University.

I have known Andrew Hague for many years. He has given me access to his files so that I can review CellSonic's progress on cancer because this looks to be the breakthrough that the world has been waiting for. Without drugs, without side effects and without any suffering inflicted on the patient the cancer appears to be cured in a matter of days.

For forty years kidney stones have been hit by pressure waves from lithotripters without reports of side effects. Early lithotripters bore some resemblance to the CellSonic VIPP machines of today so this was guidance on how healthy tissues respond and the observation was that no harm is done. Over many years with millions of patients in hospitals the world over this is good evidence of safety - no cancers were reported after non-invasive removal of kidney stones. Thus, lithotripsy does not cause cancer.

The next question is whether an existing cancer can be spread by being targeted. With millions of kidney patients it is certain that some would have had small cancers without knowing. If cancers had erupted and spread after treatment it would certainly have been reported. That is never was reported after millions of treatments is evidence.

The hypothesis about pressure killing cancer is interesting. The authors clearly are unaware of CellSonic technology but conceive of pressure doing what they already understood could be done with heat or cold.

The first patient was a distributor for CellSonic in Bangladesh. He had deteriorated despite receiving treatments that were supposed to help and he wanted to be treated by CellSonic. On 4th November 2016 he was admitted to Sai Lee Hospital, Boravili, and Mumbai to be treated with CellSonic VIPP under the supervision of Dr. Manoj Gupta. He came with complete tests carried out in Bangladesh and before treatment in India more tests were done in the usual way to be sure they knew the condition of the patient. I have read all the reports which are too many to include in this review but be it sufficient to say that they were what you would get on any cancer patient. Cancer that began in the prostate had spread. From the reports the following protocol was devised:

[Figure 1, Figure 2]

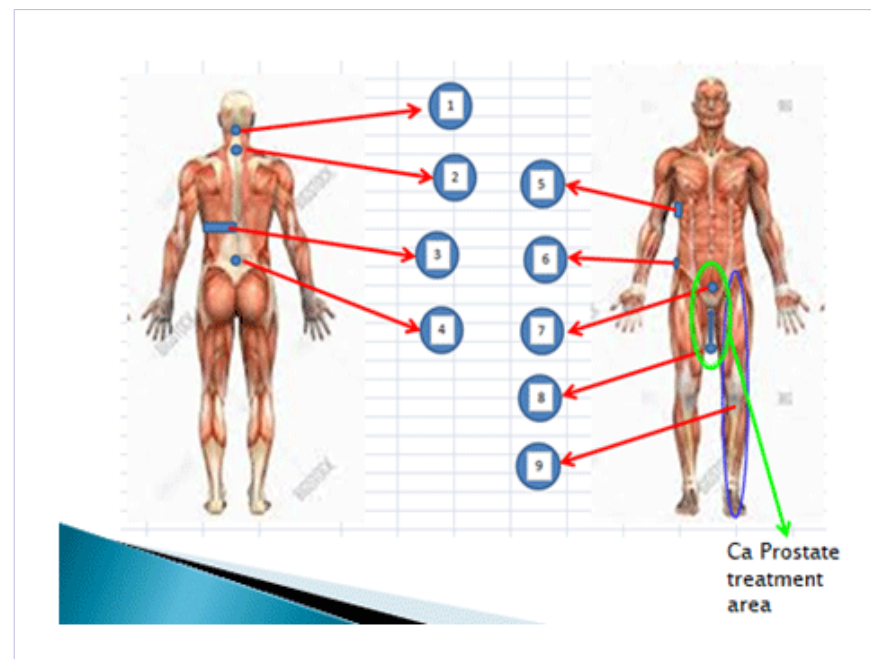

Figure 1

TREATMENT PROTOCOL :

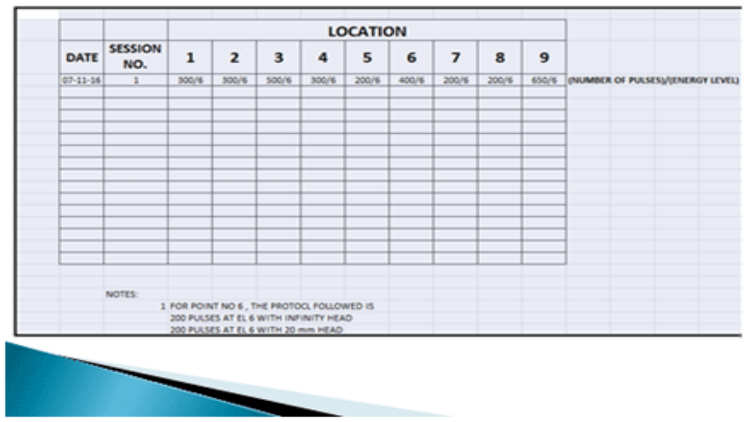

Figure 2 
On the third day, after a second treatment, the patient declared himself cured. That assessment is evidence. Although it cannot be measured, coming from a man with medical training who had been in pain for over a year to say he is cured of cancer so quickly is significant. Tests were then done by the hospital's usual methods. I have seen the results and they confirm that the patient was cured of cancer.

At the same time another CellSonic customer was treating patients for cancer and using stem cell techniques. This company, Stem RX in Mumbai, also reported success curing cancer. How much of the cure is attributable to stem cells and how much to the VIPP (Very Intense Pressure Pulses) of CellSonic is not clear so as it was said to work I am including it in this review.

Naturally these reports were received by CellSonic customers worldwide. In Peru Dr Dario Rodriguez was sent a lady patient with cancer in the spine. She arrived at the clinic in a wheelchair Dr Rodriguez treated her and she left the clinic walking and was later reported having gone to New York for a holiday. That is over a year ago and apparently she considers herself cured, is out of pain and has not sought further medical help. Dr Rodriguez had another patient with similar results and instead of encouraging him it has left him hesitant. As a trained and senior doctor he does not expect to perform miracles where a patient suddenly gets up and walks pain free after months and years of a debilitating disease. He did not know what was going on and fearing ridicule from his peers has been scared to claim he has found a cure. In my view as a senior surgeon, this is important information for the medical community. We have to accept facts as they are and not miracles. When the information comes from reputable doctors, we have to take notice.

Meanwhile the team under Dr Gupta in Mumbai performed more treatments with similar successes on cancers in various locations having spread from their origin.

In the summer of 2016, Andrew Hague, the owner of CellSonic Limited, gave a lecture to Bradford University about the CellSonic technology. From that introduction came collaboration on cancer to further the development at the university of cancer diagnosis non-invasively and without drugs. This brought Professor Simon Shepherd and Professor Raed A. Abd-Alhameed, medical engineering experts, into the team. I have met them and seen the work in their laboratory and confirm that they are getting results that will turn the understanding of cancer upside down.

The conventional approach to cancer is to kill tumors by toxins, radiation or surgical removal. All three methods have dangerous side effects and often make a bad situation worse. The advised view now is that cancer has polarity which is negative outside and positive inside, usually -70 micro volt. The CellSonic pulses will change the cancer cell's polarity stopping further replication affecting the RNA and TPAs by depleting the mitochondria.

This accords with the statement made by Dr Johannes Holfeld of Innsbruck University that he had been able with pressure to stop the replication of unhealthy cells immediately and make them replicate only healthy cells. That was in 2013 and I have been unable to elicit further information from Dr Holfeld although we do know that one of his sponsors is trying to cure cancer in combination with chemotherapy.

Professor Andrew Hague gave a clear protocol for using CellSonic on cancer and it stipulates that there must be no chemotherapy in the patient. At least four weeks must elapse since an injection if CellSonic is to be used. An interesting report came from Belgium where a senior oncologist treated patients with CellSonic and found no change. Prof Hague investigated and found that all the patients were full of chemotherapy. Their immune system was therefore non-functioning. Dr Mahajan at Stem RX and Dr Busch at Dermateam in Switzerland both confirmed that blocking the immune system with chemotherapy is both dangerous and futile. This negative result highlighted the positive side to the CellSonic treatment; done correctly it works.

The Belgian oncologist said that it is standard procedure to give cancer patients as much as they can take. He had applied four times as many pulses as recommended. By ignoring tested advice he had proved the danger of deviating from the correct CellSonic protocol.

The difference in approach is important to understand how cancer is cured. Killing cells has an emotional appeal; kill it before it kills you. Unfortunately, this is not the body's way. Cancer is the replication of mutant cells instead of the replication of healthy cells. The differences between the two classes of cells, and there are only two classes in the whole body, is that a cancer cell has a permittivity of 9.6 on the scale adopted at Bradford University with healthy cells showing 3.2. On the same scale air is 1 . The cure is to switch the polarity of the cell to change the permittivity from 9.6 to 3.2. In other words, the behaviour of the cell is changed and changed immediately as the Innsbruck University research in 2013 found.

After switching off the replication of mutant cells, the malignant tumor remains as a benign tumor. What to do with it depends on the size of the tumor and health of the patient. The patient will be damaged by the cancer and weak after the ordeal. The recovery has been the specialty of Professor Ramesh Chouhan of Jawaharlal Institute of Post Graduate Medical Education \& Research in Hyderabad. He has treated many cancer patients with CellSonic VIPP and places great importance on the vegetarian diet, meditation, exercise and positive mental outlook to ensure a good recovery. No drugs are used. The testing is by CTC (Circulating Tumor Cells) liquid biopsy which he considers the best available. It is interesting how a conventional oncologist when seeing a scanned image compares what he sees with the CTC report stating no cancer present; they do not understand.

From the reports I have seen, about a hundred patients have been cured of cancer by CellSonic. There have been no failures. A success rate of $100 \%$ on cancers in all areas of the body is a result that cannot be ignored especially as there have been no side effects, the results are quick and most important of all the patients like it; they are cured easily.

More doctors are now aware of CellSonic healing wounds and 
bones and will ask the same questions I asked about safety and how it works. What it does is obvious. Use the tests you always use and check the blood diagnoses, not just the visual images. How it works will keep the researchers occupied for years and that does not matter. There are dying patients who need help and now they have it. Use it and accept that it is safe. Keep your own records and publish them to help us understand the breakthrough as I am doing in my own practice. [Figure 3]

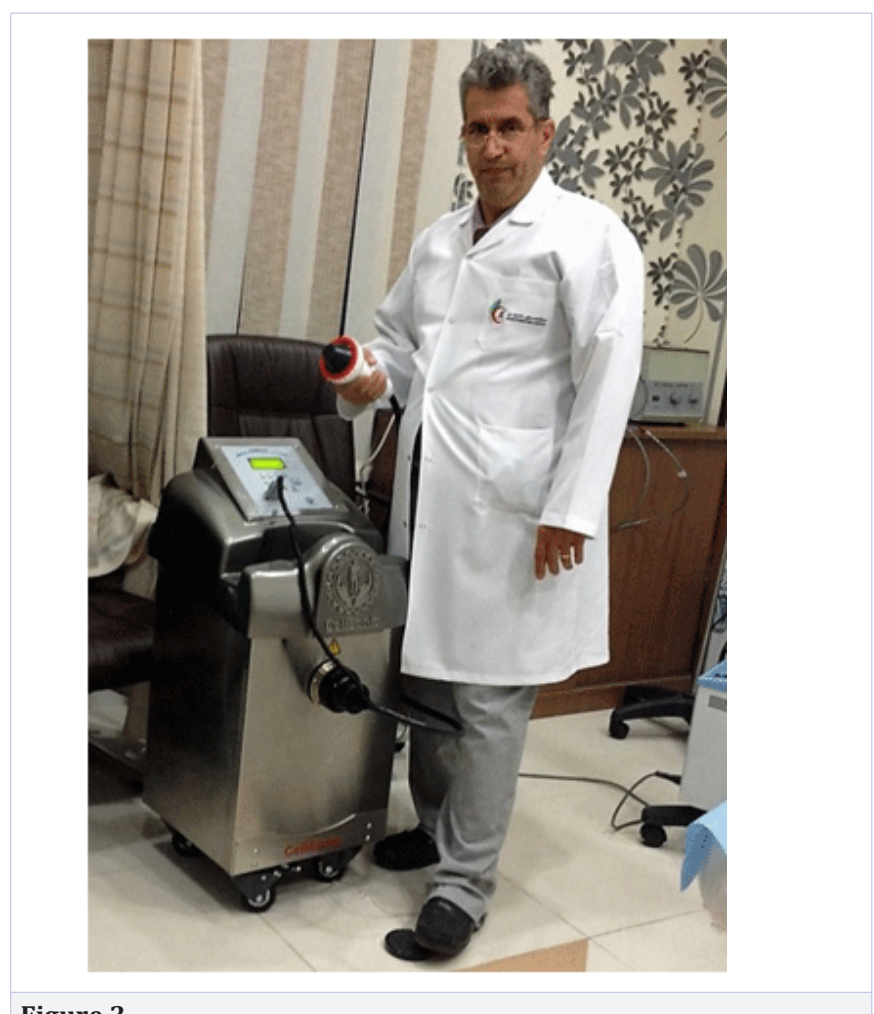

Figure 3 